\title{
Hipertensão Arterial Sistêmica Secundária
}

Coordenador:

Celso Amodeo (SP)

\section{Secretário:}

Armando da Rocha

Nogueira (RJ)

\section{Participantes:}

Adelaide A. Pereira (SP)

Antonio Carlos

Cordeiro Jr. (SP)

Armando Nogueira (RJ)

Eduardo Pimenta

(SP - Austrália)

Flávio Antonio Borelli

(SP)

José Gastão Rocha

Carvalho (PR)

Luciano Ferreira Drager

(SP)

Pedro Jabur (SP)

Rogério A. Mulinari

(PR)
A hipertensão arterial sistêmica secundária (HAS-S) tem prevalência de 3\% a 5\%. Antes de se investigarem causas secundárias de HAS devem-se excluir:

- medida inadequada da PA;

- hipertensão do avental branco;

- tratamento inadequado;

- não adesão ao tratamento;

- progressão das lesões nos órgãosalvos da hipertensão;

- presença de comorbidades;

- interação com medicamentos.

As situações em que se deve investigar a possibilidade de HAS-S estão na Tabela 1. A seguir, serão discutidas as principais causas de HAS-S.

\section{Hiperaldosteronismo Primário (HAP)}

É um grupo de doenças nas quais a produção de aldosterona está aumentada de uma forma relativamente autônoma em relação ao sistema renina-angiotensina, não sendo supressível por sobrecarga salina ou bloqueio do SRAA. Essas doenças são representadas, principalmente, pela hiperplasia bilateral das adrenais (HBA) e pelo adenoma unilateral produtor de aldosterona (APA), mas podem ser causadas por hiperplasia adrenal unilateral, carcinoma adrenal, tumores extra-adrenais produtores de aldosterona ou ter origem genética. A prevalência do HAP na população de hipertensos, de modo geral, está em torno de $6,1 \%$, mas pode variar de $3 \%$ a $22 \%$, sendo mais alta nos hipertensos em estágio 3 e/ou de difícil controle. Sabe-se que a prevalência de hipopotassemia no hiperaldosteronismo primário varia de $9 \%$ a $37 \% .{ }^{1-3}$ As etapas para rastreamento, confirmação de diagnóstico e tratamento estão descritas na Figura 1.

\section{Feocromocitoma e Paragangliomas}

São tumores de células argentafins que se localizam na medula adrenal (feocromocitomas) ou em regiões extra-adrenais (paragangliomas), que, em geral, produzem catecolaminas e se associam a quadro clínico de HAS paroxística $30 \%$ dos casos) ou sustentada com ou sem paroxismos $(50 \%$ a $60 \%)$. O diagnóstico laboratorial do tumor é baseado nas dosagens de catecolaminas e seus metabólitos no sangue e na urina (Tabela 2 ).

Para o diagnóstico topográfico dos tumores e de metástases, os métodos de imagens recomendados são: tomografia computadorizada (TC) e ressonância nuclear magnética (RNM), ambas com sensibilidade próxima a $100 \%$ para tumores adrenais. Entretanto, a RNM mostra-se superior na identificação dos paragangliomas. O mapeamento de corpo inteiro com metaiodobenzilguanidina (MIBG) tem sensibilidade de $56 \%$ para os tumores malignos a $85 \%$ para os tumores benignos, com especificidade aproximada de $100 \%$.

Octreoscan, mapeamento ósseo e tomografia por emissão de pósitrons (PET) com diferentes marcadores podem ser decisivos quando os exames de localização anteriormente citados são negativos ou então na investigação de doença maligna. ${ }^{4}$ O tratamento preferencial é o cirúrgico.

No tratamento clínico, medicamentoso, pré-operatório ou crônico são usados alfabloqueadores como prazosina ou doxazocina, combinados ou não com outros agentes, como inibidores da ECA, bloqueadores dos canais de cálcio e betabloqueadores (utilizados apenas após alfabloqueio efetivo). Em tumores inoperáveis ou no preparo pré-operatório pode ser utilizada, quando disponível, droga 


\section{Tabela 1 ACHADOS QUE SUGEREM HIPERTENSÃO ARTERIAL SECUNDÁRIA}

\begin{tabular}{lll} 
Achados & Suspeita diagnóstica & Estudos diagnósticos adicionais \\
\hline $\begin{array}{l}\text { Ronco, sonolência diurna, síndrome } \\
\text { metabólica (ver página 48) }\end{array}$ & Apneia obstrutiva do sono & Polissonografia \\
$\begin{array}{l}\text { Hipertensão resistente ao tratamento e/ } \\
\text { ou com hipocalemia e/ou com nódulo }\end{array}$ & Hiperaldosteronismo primário & $\begin{array}{l}\text { Relação aldosterona/atividade de renina } \\
\text { plasmática }\end{array}$ \\
\hline
\end{tabular}

adrena

Insuficiência renal, doença

Doença renal parenquimatosa Taxa de filtração glomerular, ultrassonogracardiovascular aterosclerótica, edema, ureia elevada, creatinina elevada, proteinúria/hematúria

Sopro sistólico/diastólico abdominal, Doença renovascular edema pulmonar súbito, alteração de função renal por medicamentos que bloqueiam o sistema reninaangiotensina

\begin{tabular}{|c|c|c|}
\hline $\begin{array}{l}\text { Uso de simpaticomiméticos, } \\
\text { perioperatório, estresse agudo, } \\
\text { taquicardia }\end{array}$ & Catecolaminas em excesso & $\begin{array}{l}\text { Confirmar normotensão em ausência de } \\
\text { catecolaminas }\end{array}$ \\
\hline $\begin{array}{l}\text { Pulsos em femorais reduzidos ou } \\
\text { retardados, raios-x de tórax anormal }\end{array}$ & Coarctação da aorta & $\begin{array}{l}\text { Doppler ou tomografia computadorizada de } \\
\text { aorta }\end{array}$ \\
\hline $\begin{array}{l}\text { Ganho de peso, fadiga, fraqueza, } \\
\text { hirsutismo, amenorreia, face em } \\
\text { "lua cheia", "corcova" dorsal, estrias } \\
\text { purpúricas, obesidade central, } \\
\text { hipopotassemia }\end{array}$ & Síndrome de Cushing & $\begin{array}{l}\text { Determinações: cortisol urinário de } 24 \\
\text { horas e cortisol matinal (8h) basal e } 8 \\
\text { horas após administração de } 1 \mathrm{mg} \text { de } \\
\text { dexametasona às } 24 \mathrm{~h} \text {. }\end{array}$ \\
\hline $\begin{array}{l}\text { Hipertensão paroxística com cefaleia, } \\
\text { sudorese e palpitações }\end{array}$ & Feocromocitoma & $\begin{array}{l}\text { Determinações de catecolaminas e seus } \\
\text { metabólitos em sangue e urina }\end{array}$ \\
\hline $\begin{array}{l}\text { Fadiga, ganho de peso, perda de } \\
\text { cabelo, hipertensão diastólica, fraqueza } \\
\text { muscular }\end{array}$ & Hipotireoidismo & Determinações de T4 livre e TSH \\
\hline $\begin{array}{l}\text { Intolerância ao calor, perda de peso, } \\
\text { palpitações, hipertensão sistólica, } \\
\text { exoftalmia, tremores, taquicardia }\end{array}$ & Hipertireoidismo & Determinações de T4 livre e TSH \\
\hline $\begin{array}{l}\text { Litíase urinária, osteoporose, depressão, } \\
\text { letargia, fraqueza muscular }\end{array}$ & Hiperparatireoidismo & Determinações de cálcio sérico e PTH \\
\hline $\begin{array}{l}\text { Cefaleias, fadiga, problemas visuais, } \\
\text { aumento de mãos, pés e língua }\end{array}$ & Acromegalia & $\begin{array}{l}\text { Determinação IGF-1 e de hormônio do cres- } \\
\text { cimento basal e durante teste de tolerância } \\
\text { oral à glicose }\end{array}$ \\
\hline
\end{tabular}
fia renal, pesquisa de microalbuminúria ou proteinúria

Doença renovascular Angiografia por ressonância magnética ou tomografia computadorizada, ultrassonografia com Doppler, renograma, arteriografia renal

\section{Tabela 2 PERCENTUAIS DE SENSIBILIDADE E ESPECIFICIDADE COM OS RESPECTIVOS INTERVALOS DE CONFIANÇA DOS TESTES BIOQUÍMICOS PARA DIAGNÓSTICO DO FEOCROMOCITOMA}

\begin{tabular}{lcc} 
Teste bioquímico & Sensibilidade & Especificidade \\
\hline Plasma & $99(96-100)$ & $89(87-92)$ \\
Metanefrinas livres & $84(78-89)$ & $81(78-84)$ \\
Catecolaminas & & $69(64-72)$ \\
\hline Urina & $97(92-99)$ & $88(85-91)$ \\
Metanefrinas fracionadas & $86(80-91)$ & $93(89-97)$ \\
Catecolaminas & $77(68-85)$ & $95(93-97)$ \\
\hline Metanefrinas - Total & $64(55-71)$ & \\
\hline
\end{tabular}


Figura 1. Fluxograma para investigação e tratamento do hiperaldosteronismo primário (HAP)

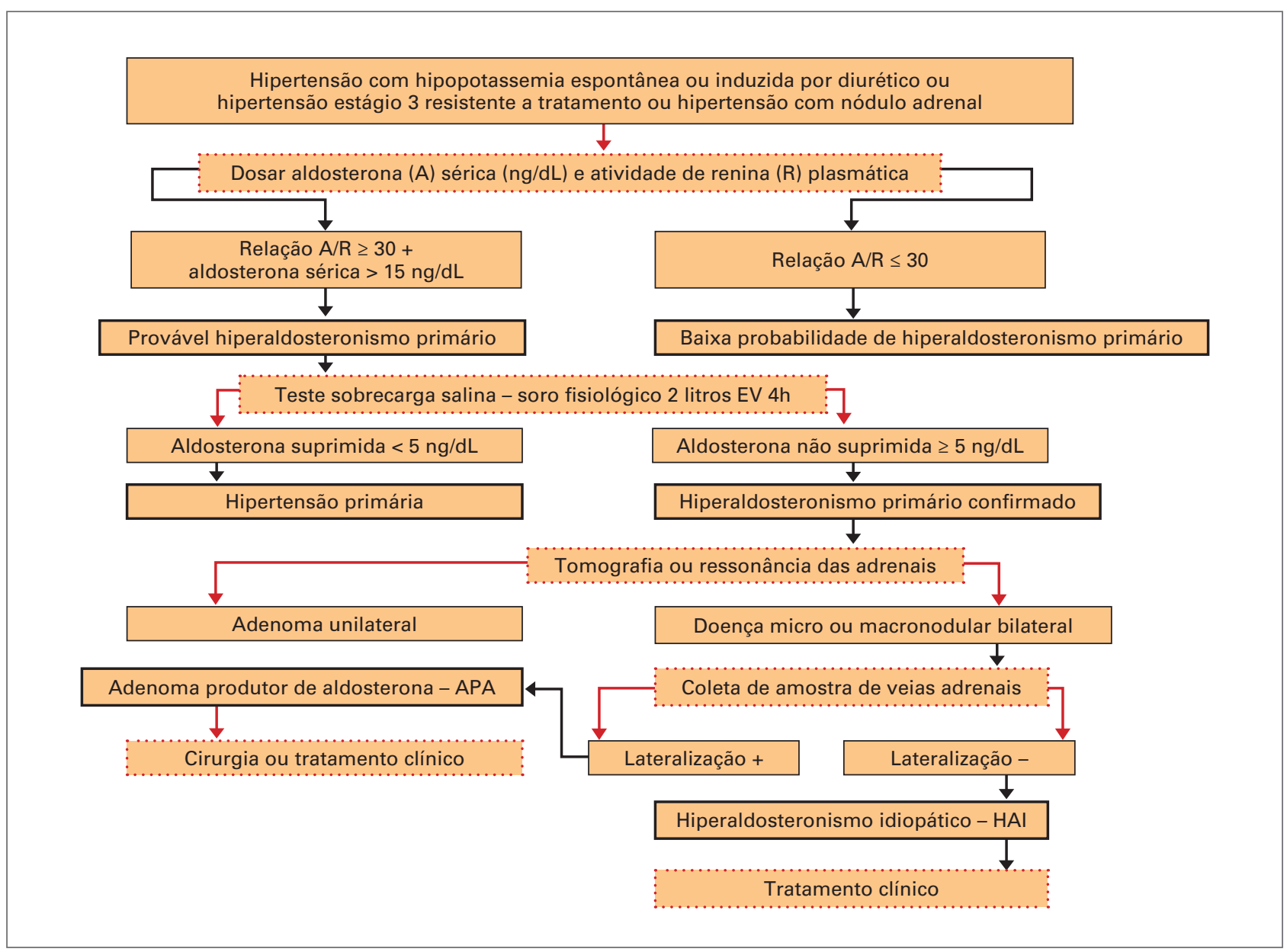

inibidora da síntese de catecolaminas: a alfametiltirosina $\left(\right.$ Demser $\left.^{\circledR}\right)$. Para a intervenção cirúrgica, recomenda-se controle da PA com o uso endovenoso de nitroprussiato de sódio e reposição volêmica, se necessária. Em crises agudas e durante a cirurgia, nitroprussiato de sódio e antiarrítmicos endovenosos (xilocaína) são agentes frequentemente utilizados. . $^{5}$

O seguimento clínico, bioquímico e radiológico contínuo dos pacientes é essencial para a detecção de recorrências ou metástases na doença maligna e de um segundo tumor nas síndromes familiares.

\section{HipotiREoIDISMO}

A prevalência de hipotireoidismo no Brasil não é conhecida com precisão, mas estima-se que esteja entre $5 \%$ e $10 \%$, sendo mais frequente no sexo feminino. ${ }^{7} \mathrm{~A}$ hipertensão arterial sistêmica ocorre em $20 \%$ dos pacientes com hipotireoidismo. ${ }^{8-10} \mathrm{O}$ diagnóstico precoce é feito pela dosagem de TSH aumentado e confirmado com a diminuição gradativa de T4 livre. Achados clínicos mais comuns são ganhos de peso, queda de cabelo e fraqueza muscular.
O tratamento deve ser iniciado com a reposição de hormônio tireoidiano e caso persista a HAS está indicado o uso de medicamentos anti-hipertensivos (C).

\section{Hipertireoidismo}

A prevalência de hipertireoidismo no Brasil está em torno de $6,5 \%,{ }^{7}$ mas pelo excesso de iodo na dieta provavelmente essa doença deve estar se tornando mais prevalente.

A apresentação clínica mimetiza um quadro hiperadrenérgico. Os principais sintomas incluem palpitação, tremor, fadiga, intolerância ao calor, hiperatividade, perda de peso e labilidade emocional. Os sinais mais importantes são: exoftalmia, hipertermia, reflexos exaltados, primeira bulha com acentuação do componente pulmonar e pele úmida. ${ }^{11} \mathrm{O}$ diagnóstico é confirmado por nível baixo de TSH e elevado de T4 livre. O tratamento é geralmente acompanhado por normalização da PA. Betabloqueadores são a primeira escolha para controlar os sintomas adrenérgicos $(\mathrm{C})$. 


\section{HiperPARATIREOIDISMO}

O hiperparatireoidismo primário pode ser devido a um adenoma ou a hiperplasia da glândula paratireoide. O hiperparatireoidismo secundário geralmente surge em estágios avançados de insuficiência renal crônica. Existe ainda o pseudo-hiperparatireoidismo, que é devido a uma resistência à ação do paratormônio. Em todas essas situações é frequente HAS com prevalências de $10 \%$ a $70 \%$ em hiperparatireoidismo primário e $40 \%-50 \%$ em pseudo-hiperparatireoidismo. As causas que levam ao aumento da PA são multifatoriais. O quadro clínico manifesta-se, geralmente, por história de litíase renal, osteoporose, depressão, letargia e fraqueza muscular. O diagnóstico é feito pela dosagem de cálcio e PTH. A correção cirúrgica do hiperparatireoidismo reduz a PA dos hipertensos e não modifica a PA nos normotensos. ${ }^{12}$

\section{Síndrome de Cushing}

Hipertensão arterial sistêmica com obesidade central, fascies em lua cheia, pletora, fraqueza muscular, cansaço fácil, hirsutismo, estrias abdominais e distúrbios emocionais levantam a suspeita clínica de HAS secundária à síndrome de Cushing. Essa síndrome é devida ao excesso de cortisol, que aumenta a retenção de sódio e água promovendo expansão de volume, sendo esse fato um dos fatores da síndrome relacionados com o aparecimento de HAS em cerca de $80 \%$ dos casos. A causa do excesso de cortisol pode ser exógena, devido à administração de glicocorticoides ou do hormônio adrenocorticotrópico (ACTH), ou endógena, por excesso de produção de cortisol ou de ACTH. Teste de supressão com dexametasona e medida do cortisol livre na urina de $24 \mathrm{~h}$ são os exames recomendados para a investigação para fins diagnósticos.

A escolha e o sucesso do tratamento dependem do diagnóstico correto e da instituição do tratamento adequado. O tratamento de escolha da síndrome de Cushing é a ressecção cirúrgica da pituitária, da fonte ectópica de ACTH ou, em alguns casos, a remoção do tumor adrenocortical produtor de cortisol. Para o controle da PA é fundamental o controle hidrossalino com dieta e diuréticos, bem como associação com outras classes de medicamentos, como os inibidores da angiotensina II, os antagonistas dos canais de cálcio e os simpatolíticos centrais. ${ }^{13}$

\section{Acromegalia}

A grande maioria dos casos de acromegalia decorre do excesso de hormônio de crescimento $(\mathrm{GH})$ produzido por um adenoma da glândula pituitária. Outras formas de acromegalia são liberações do GH em excesso devido a um tumor hipotalâmico ou carcinoide localizado em outras áreas.

As alterações estruturais e funcionais da acromegalia respondem bem ao controle dos níveis sanguíneos de GH. Em muitos casos há cura com controle da PA, particularmente quando o diagnóstico e o tratamento da acromegalia são realizados precocemente. ${ }^{14}$

\section{Coarctação da Aorta ${ }^{15}$}

A coarctação da aorta (CoAo) é causa de HAS-S encontrada especialmente em crianças e adultos jovens, sendo a quarta causa mais frequente de cardiopatia congênita, e corresponde a $7 \%$ das doenças cardíacas inatas. É mais prevalente no sexo masculino, requerendo tratamento cirúrgico no primeiro ano de vida.

Pode ocorrer em qualquer local da aorta, embora seja mais comum logo após a origem da subclávia esquerda. É muito importante o diagnóstico precoce, pois há uma relação inversa entre o tempo de exposição à HAS e a sua reversão após a correção. O exame físico revela HAS em membros superiores com uma pressão arterial sistólica pelo menos $10 \mathrm{mmHg}$ maior na artéria braquial em relação à artéria poplítea. A ausência ou diminuição dos pulsos pediosos pode também representar indício da CoAo. A ausculta pode revelar sopro sistólico interescapular proveniente do local da coarctação e também sopro sistólico amplo em crescendo-decrescendo em toda a parede torácica por causa da dilatação das artérias intercostais colaterais.

O tratamento da CoAo é sempre intervencionista, podendo ser realizado por procedimento endovascular, em indivíduos mais jovens ou em crianças, ou cirurgia, nos casos de hipoplasia do arco aórtico e/ou necessidade de ressecção da coarctação. A resposta sobre a PA ao tratamento intervencionista da CoAo depende, em grande parte, da duração da hipertensão no período pré-operatório e da idade do paciente. Há cura da HAS em até $50 \%$ dos pacientes, mas pode recorrer tardiamente, especialmente se a intervenção foi feita em idades mais avançadas.

\section{Hipertensão Renovascular}

A hipertensão renovascular (HR) é definida como HAS decorrente de uma isquemia renal, geralmente causada por uma lesão obstrutiva parcial ou completa de uma ou ambas as artérias renais. Sua prevalência atinge 5\% dos pacientes com HAS. ${ }^{16,17-19}$ 
A HR pode ser causada por aterosclerose, a causa mais comum, com prevalência em torno de $90 \%$, ou displasia fibromuscular. ${ }^{20,21}$ A estenose aterosclerótica de artéria renal é geralmente progressiva. Cerca de $40 \%$ das obstruções arteriais acima de $75 \%$ evoluem para obstrução total entre um a cinco anos..$^{22-25}$

A displasia fibromuscular, por sua vez, é mais frequentemente encontrada em mulheres jovens de cor branca. Entre os vários tipos de lesões fibrodisplásicas a mais comum é aquela que envolve a camada média da parede do vaso. Geralmente seu acometimento é bilateral envolvendo as porções distais da artéria renal.

\section{DiAgnóstico}

Devido à baixa incidência de HR em pacientes com hipertensão não complicada, o rastreamento aplicado a todos os indivíduos não é custo-efetivo nessas condições. ${ }^{25}$ Algumas características clínicas (Tabela 3) chamam a atenção para a possibilidade de hipertensão renovascular.

Os métodos disponíveis e mais comumente utilizados para o diagnóstico de HR apresentam sensibilidade e especificidade diferentes (Tabela 4) (nível de evidência B).

\section{Tabela 3 CARACTERÍsticAs SUGESTIVAS DE HIPERTENSÃO RENOVASCULAR}

Início abrupto da hipertensão arterial, antes dos 30 ou após os 50 anos

Hipertensão estágio II ou III, acelerada ou maligna

Hipertensão refratária à múltipla terapia

Hipertensão estágio II ou III na presença de aterosclerose difusa

Presença de sopro epigástrico sistólico/diastólico

Hipertensão estágio II ou III com insuficiência renal sem explicação

Azotemia significativa induzida por inibidor da enzima conversora da angiotensina ou por bloqueador do receptor da angiotensina

Assimetria no tamanho renal

Edema pulmonar sem causa aparente em paciente com hipertensão

\begin{tabular}{|c|c|c|c|}
\hline \multirow{2}{*}{$\begin{array}{r}\text { Tabela } 4 \\
\text { Tipo de teste }\end{array}$} & \multicolumn{3}{|c|}{$\begin{array}{l}\text { TESTES PARA DETECÇÃO DE HIPERTENSÃO } \\
\text { RENOVASCULAR }\end{array}$} \\
\hline & & $\begin{array}{c}\text { Sensibilidade } \\
(\%)\end{array}$ & $\begin{array}{c}\text { Especificidade } \\
(\%)\end{array}$ \\
\hline \multicolumn{2}{|c|}{$\begin{array}{l}\text { Cintilografia com } \\
\text { captopril }\end{array}$} & $92-94$ & $95-75$ \\
\hline \multicolumn{2}{|c|}{ Ultrassom com Doppler } & $84-91$ & $95-97$ \\
\hline \multicolumn{2}{|c|}{ Angiografia digital } & 88 & 90 \\
\hline \multicolumn{2}{|c|}{ Angiorressonância* } & $90-95$ & 95 \\
\hline
\end{tabular}

* Na identificação de estenoses da artéria renal acima de 50\%.

\section{TRATAMENTO}

Três são as possibilidades terapêuticas na HR: medicamentosa, dilatação da estenose da artéria renal com ou sem implante de stents no vaso acometido e abordagem cirúrgica por revascularização ou autotransplante.

\section{Medicamentoso}

O tratamento medicamentoso é uma opção terapêutica bastante aceitável na ausência de evidências sugestivas de que a estenose da artéria renal é causadora de HAS ou isquemia renal. A maior parte dos pacientes com estenose de artéria renal é formada por indivíduos com HAS primária preexistente e doença aterosclerótica concomitante. Não existe teste que determine se há uma relação causal entre estenose da artéria renal e hipertensão arterial. O estudo CORAL (Cardiovascular Outcomes in Renal Atherosclerotic Lesions), ainda em andamento, tem por objetivo responder qual a abordagem mais apropriada para os casos de HR de causa aterosclerótica. ${ }^{26}$

Como a HAS secundária à estenose da artéria renal pode ser dependente da ativação do SRAA, o uso de medicamentos que o bloqueiem, como os inibidores da enzima conversora da angiotensina (nível de evidência A) e os bloqueadores dos receptores $\mathrm{AT}_{1} \mathrm{da}$ angiotensina II (nível de evidência B), pode ser especialmente eficaz. ${ }^{27}$ Contudo, esses medicamentos são contraindicados em pacientes com estenose de artéria renal bilateral ou unilateral em rim único. No mesmo nível de evidência em que estão os inibidores da enzima conversora da angiotensina estão os antagonistas dos canais de cálcio (nível de evidência A). ${ }^{28,29}$

\section{CIRURGIA X PROCEDIMENTO PERCUTÂNEO}

Considera-se estenose significativa de artéria renal a que oclui pelo menos $60 \%$ da luz do vaso estimada visualmente com gradiente de lesão maior que $20 \mathrm{mmHg}$ ou gradiente médio maior que $10 \mathrm{mmHg} .{ }^{30}$

Pacientes com lesões fibrodisplásicas e aparecimento recente de HAS tendem a se beneficiar mais com a correção da estenose, podendo até mesmo evoluir para a normalização da PA..$^{31,32}$ Pacientes com lesões ateroscleróticas e longa história de HAS podem apresentar pouca ou nenhuma melhora após o procedimento..$^{33-36}$ Podem ocorrer complicações em decorrência da intervenção percutânea para correção da estenose de artéria renal. A Tabela 5 mostra as possíveis complicações em decorrência da angioplastia.

\section{Síndrome da Apneia e Hipopneia Obstrutiva Do SONO (SAHOS)}

A SAHOS é definida como a obstrução recorrente das vias aéreas superiores durante o sono, resultando em 
Tabela 5 COMPLICAÇÕES POSSIVEIS DA REVASCULARIZAÇÃO PERCUTÂNEA

Formação de hematomas

Hemorragias

Infecção

Formação de pseudoaneurisma

Formação de fístula arteriovenosa

Nefropatia induzida por contraste

Insuficiência renal por microembolização de cristais de colesterol

Embolia para membros inferiores ou mesentério

Perfuração da artéria renal

Óbito (raramente)

períodos de apneia, hipopneia, dessaturação de oxiemoglobina e despertares frequentes com alteração da arquitetura do sono. ${ }^{36}$ Diversas evidências clínicas e experimentais sugerem que a SAHOS está relacionada ao desenvolvimento de HAS independentemente da obesidade. ${ }^{37-41} \mathrm{O}$ conjunto dessas e de outras evidências fez com que fosse incluída, em 2003, como uma causa de HAS-S. ${ }^{42}$ Estima-se que a prevalência da SAHOS em pacientes com HAS e hipertensão arterial refratária gire em torno de $30 \%$ a $56 \%$ e $71 \%$ a $82 \%$ respectivamente..$^{43-46}$ Evidências sugerem também que a SAHOS pode contribuir para o surgimento de lesões de órgãos-alvo ${ }^{47}$ e de aceleração do processo de aterosclerose nos pacientes hipertensos. ${ }^{68}$ No entanto, a falta de diagnóstico de SAHOS é ainda muito frequente. ${ }^{44,49,50}$ Embora os mecanismos precisos pelos quais promova o aumento persistente da $\mathrm{PA}$, não somente durante o período de sono, não sejam totalmente conhecidos, alguns importantes componentes que contribuem diretamente para o remodelamento vascular já foram descritos. Entre eles incluem-se ativação simpática, diminuição da sensibilidade dos barorreceptores, aumento de marcadores inflamatórios, espécies reativas de oxigênio, endotelina-1, além de disfunção endotelial, dentre outros. ${ }^{51}$

A suspeita clínica deve ser realizada especialmente se o paciente tiver diagnóstico concomitante de síndrome metabólica, pois esta condição mostrou ser uma excelente preditora de AOS em pacientes com HAS. ${ }^{44}$ Devese, também, considerar essa possibilidade em pacientes hipertensos entre 40 e 70 anos, sobretudo se houver alta probabilidade estimada pela aplicação do questionário de Berlin ${ }^{51}$ ou quando o paciente apresentar alterações no padrão de descenso fisiológico do sono..$^{53}$

O diagnóstico é confirmado pelo achado de cinco ou mais episódios de apneia e/ou hipopneia por hora - índice de apneia-hipopneia - na polissonografia. ${ }^{37}$
Para o tratamento da SAHOS, considerar sempre a perda de peso, particularmente nos indivíduos com HAS associada a sobrepeso ou obesidade. ${ }^{54} \mathrm{O}$ tratamento de escolha para os casos moderados e graves (índice de apneia-hipopneia $\geq 15$ eventos por hora) é o uso da pressão positiva contínua em vias aéreas superiores (CPAP) durante o sono. ${ }^{52}$ Esse tratamento parece resultar em reduções da PA em hipertensos não controlados e especialmente em hipertensos refratários. ${ }^{55-58}$ No que diz respeito ao tratamento antihipertensivo medicamentoso, não há, até o momento, evidências de que uma classe anti-hipertensiva seja superior a outra. ${ }^{58}$

\section{Doença Renal Crônica (DRC)}

A associação entre HAS e DRC é bem conhecida, tendo em vista que a doença renal é de longe a maior causa de HAS secundária. ${ }^{59-62}$ Sua prevalência é bastante elevada em pacientes com doença renal, situando-se entre $60 \%-100 \%$, de acordo com o tipo de população estudada. ${ }^{59-62}$ Entretanto, existem diferentes tipos de acometimento renal, sendo o diagnóstico causal importante para a escolha adequada do tratamento anti-hipertensivo a ser instituído. ${ }^{63,64}$

Os principais mecanismos patogênicos da HAS na DRC são a sobrecarga de volume e a maior ativação do SRAA. ${ }^{60-62,64}$ A meta a ser atingida com o tratamento da HAS em pacientes com DRC deve ser de $\mathrm{PA} \leq 130 / 80 \mathrm{mmHg}{ }^{63,65}$ (B). Todas as classes de antihipertensivos são eficazes na redução da PA nesses pacientes. ${ }^{63,65,66}$ Assim, o tratamento deve ser individualizado, tendo-se em mente a causa da DRC e a presença de doença cardiovascular preexistente. $\mathrm{O}$ tratamento anti-hipertensivo em pacientes com DRC deve visar não apenas a redução da PA, mas também a redução da proteinúria. ${ }^{63,65,66}$ Os IECAs e os BRAs II reduzem a proteinúria e a progressão da DRC independentemente da doença de base ${ }^{63}(\mathrm{~A})$.

Os inibidores diretos de renina mostraram-se efetivos na redução da PA e da albuminúria em pacientes diabéticos; contudo, pelo pequeno número de estudos, e pela não inclusão de pacientes com DRC estágios 4-5 nos estudos realizados, a importância desta classe de medicamentos nessas condições ainda não está completamente estabelecida. ${ }^{67,71}$

Tendo-se em mente que a hipervolemia é um importante determinante da elevação da PA nesses pacientes, os diuréticos devem ser incluídos na maioria dos esquemas anti-hipertensivos ${ }^{63,65,73,74}$ (A). Antagonistas dos canais de cálcio não di-hidropirimidínicos são efetivos na redução da proteinúria em 
pacientes com DRC secundária a nefropatia diabética. ${ }^{63,74-76}$ Em contrapartida, os di-hidropirimidínicos, além de terem mostrado ser menos efetivos que outras classes de drogas em reduzir a progressão da doença renal, podem também agravar a proteinúria em pacientes com DRC, diabéticos e não-diabéticos. Por esta razão, em pacientes com DRC e proteinúria, recomenda-se o uso dos ACC di-hidropirimidínicos apenas se associados a um IECA ou BRA..$^{63,77-80}$ Como é grande a prevalência de doença coronariana em pacientes com DRC, os betabloqueadores com frequência fazem parte do esquema anti-hipertensivo desta população. Nesse sentido é interessante notar que o carvedilol, especificamente, mostrou-se eficaz na redução da albuminúria em pacientes diabéticos tipo $2 .{ }^{63,81}$ Sabendose, também, que pacientes com DRC apresentam atividade aumentada do sistema nervoso simpático, os alfabloqueadores e os inibidores adrenérgicos de ação central são medicamentos que podem ser utilizados, alternativamente, em associação com outras classes de anti-hipertensivos. ${ }^{85,82}$ Por fim, antagonistas da aldosterona também se mostraram efetivos na redução da proteinúria, mesmo em pacientes que já se encontravam utilizando IECA e BRA em associação, contudo com aumento do risco de hipercalemia. ${ }^{63,83}$

Em pacientes com DRC estágio 5 em terapia dialítica, a hipervolemia tem papel preponderante na etiologia da HAS. Dessa forma, o ajuste adequado do "peso-seco" é a medida primordial para controle da PA nessa população (A)..$^{84-95}$

\section{Medicamentos e Drogas}

A Tabela 6 apresenta os medicamentos e drogas, lícitas e ilícitas, relacionadas com o desenvolvimento ou agravamento da HAS.

\section{Tabela 6 MEDICAMENTOS E DROGAS, LícITAS E ILÍCITAS, RELACIONADAS COM O DESENVOLVIMENTO OU AGRAVAMENTO DA HIPERTENSÃO ARTERIAL SISTÊMICA}

\begin{tabular}{|c|c|c|}
\hline Classe de medicamentos & Efeito sobre a PA e frequência & Ação sugerida \\
\hline \multicolumn{3}{|l|}{ Imunossupressores } \\
\hline Ciclosporina, tacrolimus & Intenso e frequente & $\begin{array}{l}\text { Inibidor de ECA e antagonista de canal } \\
\text { de cálcio (nifedipino/anlodipino). Ajustar } \\
\text { nível sérico. Reavaliar opções }\end{array}$ \\
\hline
\end{tabular}

\section{Glicocorticoides}

Anti-inflamatórios não esteroides Inibidores da ciclooxigenase $1 \mathrm{e}$ ciclooxigenase 2

Eventual, muito relevante com uso

Anorexígenos/Sacietógenos

Anfepramona e outros

Sibutramina

Vasoconstritores, incluindo derivados do ergot

Hormônios

Eritropoietina humana

Anticoncepcionais orais

Terapia de reposição estrogênica (estrogênios conjugados e estradiol)

Hormônio de crescimento (adultos)

Antidepressivos

Inibidores da monoaminoxidase

Tricíclicos

Intenso, infrequente

Variável e frequente contínuo

Observar função renal e informar efeitos adversos

Intenso e frequente

Suspensão ou redução de dose

Avaliar a redução da pressão arterial obtida com a redução de peso

Usar por período determinado
Avaliar hematócrito e dose semanal

Avaliar a substituição do método com especialista

Avaliar risco e custo-benefício

Suspensão

Abordar como crise adrenérgica Abordar como crise adrenérgica Vigiar interações medicamentosas

Drogas ilícitas e álcool

Anfetamina, cocaína e derivados

Álcool
Efeito agudo, intenso. Dosedependente

Variável e dose-dependente Muito prevalente
Abordar como crise adrenérgica

Vide tratamento não medicamentoso 


\section{REFERÊNCIAS}

1. Funder JW, Carey RM, Fardella C et al. Case detection, diagnosis and treatment of patients with primary aldosteronism: an Endocrine Society Clinical Practice Guideline. J Clin Endocrinol Metab 2008; 93:3266-3281.

2. Stowasser M. Update in primary aldosteronism. J Clin Endocrinol Metab 2009; 94:3623-3630.

3. Wiebke A. A detour guide to The Endocrine Society Clinical Practice Guideline on case detection, diagnosis and treatment of patients with primary aldosteronism. Eur J Endocrinol 2009 Oct 1. [Epub ahead of print].

4. Eisenhofer G, Siegert G, Kotzerke J, Bornstein SR, Pacak K. Current Progress and Future Challenges in the Biochemical Diagnosis and Treatment of Pheochromocytomas and Paragangliomas. Horm Metab Res 2008; 40:329-447.

5. Pacak K, Eisenhofer G, Ahlman H et al. Pheochromocytoma: recommendations for clinical practice from the First International Symposium. Nat Clin Prac Endocrinol Metab 2007; 3(2):92-102.

6. Pacak K. Approach to the patient. Preoperative Management of the Pheochromocytoma Patient. J Clin Endocrinol Metab 2007; 92:4069-4079.

7. Duarte GC, Tomimori EK, Camargo RYA et al. The prevalence of thyroid dysfunction in elderly cardiology patients with mild excessive iodine intake in the urban area of São Paulo. Clinics 2009; 64:135- 142.

8. Chiong JR, Aronow WS, Khan IA et al. Secondary hypertension: current diagnosis and treatment. Int J Cardiol 2008; 124:6-21.

9. Liu D, Jiang F, Shan Z et al. A cross-sectional survey of relationship between serum TSH level and blood pressure. J Hum Hypertens 2009; advance on line publication 25 june.

10. Dernellis J, Panaretou M. Effects of thyroid replacement therapy on arterial blood pressure in patients with hypertension and hypothyroidism. Am Heart J 2002; 143:718-724.

11. Volzke H, Ittermann T, Schmidt CO et al. Subclinical hyperthyroidism and blood pressure in a populationbased prospective cohort study. Eur J End 2009; 161:615-621.

12. Heyliger A, Tangpricha V, Weber C, Sharma J. Parathyroidectomy decreases systolic and diastolic blood pressure in hypertensive patients with primary hyperparathyroidism. Surgery 2009 Dec; 146(6):1042- 1047.

13. Magiakou MA, Smymaki P, Chrousos GP. Hypertension in Cushing's syndrome. Clin Endocrinol Metab 2006; 20:467-482.

14. Ezzat S, Foster MJ, Berchtold P, Redelmeier DA, Boerlin V, Harris AG. Acromegaly: clinical and biochemical features in 500 patients. Medicine 1994; 73:233-240.

15. Webb GD, Smallhorn J F, Therrien J et al. Congenital heart disease. In: Braunwald E, Zipes D, Libby P (eds). Heart Disease. Philadelphia: Elsevier; 2005:1532-1535.

16. Elliott WJ. Secondary hypertension: reno-vascular hypertension. In: Black \& Elliott WG (eds). Hypertension: a companion to Braunwald's heart disease. Saunders Elsevier 2007, pp 93-105. RV.

17. Uzu T, Inoue T, Fujii T et al. Prevalence and predictors of renal artery stenosis in patients with myocardial infarction. Am J Kidney Dis 1997; 29:733-738.
18. Wilms G, Marchal G, Peene P et al. The angiographic incidence of renal artery stenosis in the arteriosclerotic population. Eur J Radiol 1990; 10:195-197.

19. Swartbol P, Thorvinger BO, Parsson $\mathrm{H}$ et al. Renal artery stenosis in patients with peripheral vascular disease and its correlation to hypertension: a retrospective study. Int Angiol 1992; 11:195-199.

20. Safian RD, Textor SC. Renal-artery stenosis. N Engl J Med 2001; 344:431-442.

21. White, CJ. Catheter-based therapy for atherosclerotic renal artery stenosis. Circulation 2006; 113:1464-1473.

22. Schreiber MJ, Pohl MA, Novick AC. The natural history of atherosclerotic and fibrous renal artery disease. Urol Clin North Am 1984; 11:383-392.

23. Zierler RE, Bergelin RO, Davidson RC et al. A prospective study of disease progression in patients with atherosclerotic renal artery stenosis. Am J Hypertens 1996; 9:1055-1056.

24. Zierler RE. Screening for renal artery stenosis: is it justified? Mayo Clin Proc 2002; 77:307-308.

25. Cooper CJ, Murphy TP, Matsumoto A et al. Stent revascularization for the prevention of cardiovascular and renal events among patients with renal artery stenosis and systolic hypertension: rationale and design of the CORAL trial. Am Heart J 2006; 152:59-66.

26. Plouin P F, Chatellier G, Darne B et al. Blood pressure outcome of angioplasty in atherosclerotic renal artery stenosis: a randomized trial. Essai Multicentrique Medicaments $v s$. Angioplastie (EMMA) Study Group. Hypertension 1998; 31:823-829.

27. Hollenberg NK. Medical therapy of renovascular hypertension: efficacy and safety of captopril in 269 patients. Cardiovasc Rev Repl 1983; 4:852-876.

28. Olin JW. Role of duplex ultrasonography in screening for significant renal artery disease. Urol Clin North Am 1994; 21:215-226.

29. Luscher T F, Keller HM, Imhof HG et al. Fibromuscular hyperplasia: extension of the disease and therapeutic outcome: results of the University Hospital Zurich Cooperative Study on Fibromuscular Hyperplasia. Nephron 1986; 44 (suppl 1):109-114.

30. Schreiber MJ, Pohl MA, Novick AC. The natural history of atherosclerotic and fibrous renal artery disease. Urol Clin North Am 1984; 11:383-392.

31. Watson PS, Hadjipetrou P, Cox SV, Piemonte TC, Eisenhauer AC. Efect of renal artery stenting on renal function and size in patients with atherosclerotic renovascular disease. Circulation 2000; 102:1671-1677.

32. Nordmann AJ, Woo K, Parkes R, Logan AG. Ballon angioplasty or medical therapy for hypertensive patients with atherosclerotic renal artery stenosis? A meta-analysis of randomized controlled trials. Am J Med 2003; 114:44-50.

33. Leertouwer TC, Gussenhoven EJ, Bosch JL et al. Stent placement for renal artery stenosis: where do we stand? A meta-analysis. Radiology 2000; 216:78-85.

34. Boisclair C, Therasse E, Oliva VL et al. Treatment of renal angioplasty failure by percutaneous renal artery stenting with Palmaz stents. AJR Am J Roentgenol 1997; 168(1):245-251.

35. Stanley JC. Surgical treatment of renovascular hypertension. Am J Surg. 1997; 174(2):102- 110. 
36. Sleep-related breathing disorders in adults: recommendations for syndrome definition and measurement techniques in clinical research. The Report of an American Academy of Sleep Medicine Task Force. Sleep. 1999; 22(5):667-689.

37. Peppard PE, Young T, Palta M, Skatrud J. Prospective study of the association between sleep-disordered breathing and hypertension. N Engl J Med 2000; 342:1378-1384.

38. Nieto FJ, Young TB, Lind BK et al. Association of sleep-disordered breathing, sleep apnea, and hypertension in a large community-based study. Sleep Heart Health Study. JAMA. 2000; 283(14):1829-1836. Erratum in: JAMA 2002; 288(16):1985.

39. Lavie P, Herer P, Hoffstein V. Obstructive sleep apnoea syndrome as a risk factor for hypertension: population study. BMJ 2000; 320(7233):479-482.

40. Brooks D, Horner RL, Kozar L F, Render-Teixeira CL, Phillipson EA. Obstructive sleep apnea as a cause of systemic hypertension. Evidence from a canine model. J Clin Invest 1997; 99(1):106-109.

41. Chobanian AV, Bakris GL, Black HR et al. Joint National Committee on Prevention, Detection, Evaluation, and Treatment of High Blood Pressure. National Heart, Lung, and Blood Institute; National High Blood Pressure Education Program Coordinating Committee. Seventh report of the Joint National Committee on Prevention, Detection, Evaluation, and Treatment of High Blood Pressure. Hypertension. 2003; 42(6):1206-1252.

42. Sjöström C, Lindberg E, Elmasry A, Hägg A, Svärdsudd K, Janson C. Prevalence of sleep apnoea and snoring in hypertensive men: a population based study. Thorax 2002; 57(7):602-607.

43. Drager L F, Genta PR, Pedrosa RP et al. Characteristics and predictors of obstructive sleep apnea in patients with systemic hypertension. Am J Cardiol. 2010 (in press).

44. Gonçalves SC, Martinez D, Gus M et al. Obstructive sleep apnea and resistant hypertension: a case-control study. Chest. 2007; 132(6):1858-1862.

45. Logan AG, Perlikowski SM, Mente A et al. High prevalence of unrecognized sleep apnoea in drug-resistant hypertension. J Hypertens 2001; 19(12):2271-2277.

46. Drager L F, Bortolotto LA, Figueiredo AC, Silva BC, Krieger EM, Lorenzi-Filho G. Obstructive sleep apnea, hypertension and their interaction on arterial stiffness and heart remodeling. Chest 2007; 131:1379-1386.

47. Drager L F, Bortolotto LA, Krieger EM, Lorenzi-Filho G. Additive effects of obstructive sleep apnea and hypertension on early markers of carotid atherosclerosis. Hypertension 2009; 53(1):64-69.

48. Silverberg DS, Oksenberg A, Iaina A. Sleep related breathing disorders are common contributing factors to the production of essential hypertension but are neglected, underdiagnosed, and undertreated. Am J Hypertens 1997; 10:1319-1325.

49. Gus M, Gonçalves SC, Martinez D et al. Risk for obstructive sleep apnea by Berlin Questionnaire, but not daytime sleepiness, is associated with resistant hypertension: a casecontrol study. Am J Hypertens 2008; 21(7):832-835.

50. Bradley TD, Floras JS. Obstructive sleep apnoea and its cardiovascular consequences. Lancet 2009; 373(9657):82-93.
51. Netzer NC, Stoohs RA, Netzer CM, Clark K, Strohl K P. Using the Berlin Questionnaire to identify patients at risk for the sleep apnea syndrome. Ann Intern Med 1999; 131(7):485-491.

52. Suzuki M, Guilleminault C, Otsuka K, Shiomi T. Blood pressure "dipping" and "non-dipping" in obtructive sleep apnea syndrome patients. Sleep. 1996; 19(5):382-387.

53. Peppard PE, Young T, Palta M, Dempsey J, Skatrud J. Longitudinal study of moderate weight change and sleep-disordered breathing. JAMA. 2000; 284(23):3015-3021.

54. Andrén A, Sjöquist M, Tegelberg A. Effects on blood pressure after treatment of obstructive sleep apnoea with a mandibular advancement appliance - a three-year follow-up. J Oral Rehabil. 2009; 36(10):719-725.

55. Gotsopoulos H, Kelly JJ, Cistulli PA. Oral appliance therapy reduces blood pressure in obstructive sleep apnea: a randomized, controlled trial. Sleep 2004; 27(5):934- 941.

56. Sullivan CE, Issa FG, Berthon-Jones M, Eves L. Reversal of obstructive sleep apnoea by continuous positive airway pressure applied through the nares. Lancet 1981; 1(8225):862-865.

57. Bazzano LA, Khan Z, Reynolds K, He J. Effect of nocturnal nasal continuous positive airway pressure on blood pressure in obstructive sleep apnea. Hypertension 2007; 50:417-423.

58. Haentjens P, Van Meerhaeghe A, Moscariello A et al. The impact of continuous positive airway pressure on blood pressure in patients with obstructive sleep apnea syndrome: evidence from a meta-analysis of placebocontrolled randomized trials. Arch Intern Med 2007; 167(8):757-764.

59. Campese VM, Mitra D, Sandee D. Hypertension in renal parenchymal disease: why is it so resistant to treatment? Kidney Int 2006; 69(6):967-973.

60. Ritz E. Hypertension: the kidney is the culprit even in the absence of kidney disease. Kidney Int 2007; 71(5):371-372.

61. Siamopoulos K. Treatment of hypertension in patients with chronic renal failure. Nephrol Dial Transplant 2001; 16 (Suppl 6):46-47.

62. K/DOQI clinical practice guidelines on hypertension and antihypertensive agents in chronic kidney disease. Am J Kidney Dis 2004; 43(5 Suppl 1):S1-290.

63. Sica DD, Carl D. Pathologic basis and treatment considerations in chronic kidney disease-related hypertension. Semin Nephrol 2005; 25(4):246-251.

64. Mancia G, De Backer G, Dominiczak A et al. 2007 Guidelines for the Management of Arterial Hypertension: The Task Force for the Management of Arterial Hypertension of the European Society of Hypertension (ESH) and of the European Society of Cardiology (ESC). J Hypertens 2007; 25(6):1105-1187.

65. Khosla N, Bakris G. Lessons learned from recent hypertension trials about kidney disease. Clin J Am Soc Nephrol 2006; 1(2):229-235.

66. Bakris GL, Copley JB, Vicknair N, Sadler R, Leurgans S. Calcium channel blockers versus other antihypertensive therapies on progression of NIDDM associated nephropathy. Kidney Int 1996; 50(5):1641- 1650. 
67. Brenner BM, Cooper ME, de Zeeuw D et al. Effects of losartan on renal and cardiovascular outcomes in patients with type 2 diabetes and nephropathy. N Engl J Med 2001; 345(12):861-869.

68. Chan JC, Ko GT, Leung DH et al. Long-term effects of angiotensin-converting enzyme inhibition and metabolic control in hypertensive type 2 diabetic patients. Kidney Int 2000; 57(2):590-600.

69. Hebert LA, Bain RP, Verme D et al. Remission of nephrotic range proteinuria in type I diabetes. Collaborative Study Group. Kidney Int 1994; 46(6):1688-1693.

70. Hovind P, Rossing P, Tarnow L, Smidt UM, Parving $\mathrm{HH}$. Remission and regression in the nephropathy of type 1 diabetes when blood pressure is controlled aggressively. Kidney Int 2001; 60(1):277-283.

71. Lebovitz HE, Wiegmann TB, Cnaan A, et al. Renal protective effects of enalapril in hypertensive NIDDM: role of baseline albuminuria. Kidney Int Suppl 1994; 45:S150-155.

72. Lewis EJ, Hunsicker LG, Bain RP, Rohde RD. The effect of angiotensin-converting-enzyme inhibition on diabetic nephropathy. The Collaborative Study Group. N Engl J Med 1993; 329(20):1456-1462.

73. Lewis EJ, Hunsicker LG, Clarke WR, et al. Renoprotective effect of the angiotensin-receptor antagonist irbesartan in patients with nephropathy due to type 2 diabetes. N Engl J Med 2001; 345(12):851-860.

74. Nielsen FS, Rossing P, Gall MA, Skott P, Smidt UM, Parving HH. Impact of lisinopril and atenolol on kidney function in hypertensive NIDDM subjects with diabetic nephropathy. Diabetes 1994; 43(9):1108-1113.

75. Parving HH, Lehnert H, Brochner-Mortensen J, Gomis R, Andersen S, Arner P. The effect of irbesartan on the development of diabetic nephropathy in patients with type 2 diabetes. N Engl J Med 2001; 345(12):870-878.

76. Ravid M, Savin H, Jutrin I, Bental T, Katz B, Lishner M. Long-term stabilizing effect of angiotensin-converting enzyme inhibition on plasma creatinine and on proteinuria in normotensive type II diabetic patients. Ann Intern Med 1993; 118(8):577-581.

77. Schoolwerth AC, Sica DA, Ballermann BJ, Wilcox CS. Renal considerations in angiotensin converting enzyme inhibitor therapy: a statement for healthcare professionals from the Council on the Kidney in Cardiovascular Disease and the Council for High Blood Pressure Research of the American Heart Association. Circulation 2001; 104(16):1985-1991.

78. Viberti G, Mogensen CE, Groop LC, Pauls JF. Effect of captopril on progression to clinical proteinuria in patients with insulin-dependent diabetes mellitus and microalbuminuria. European Microalbumi-nuria Captopril Study Group. JAMA 1994; 271(4):275-279.

79. Wilmer WA, Hebert LA, Lewis EJ et al. Remission of nephrotic syndrome in type 1 diabetes: long-term follow-up of patients in the Captopril Study. Am J Kidney Dis 1999; 34(2):308-314.
80. Brown MJ. Aliskiren. Circulation 2008; 118(7):773-784. 81. Musini VM, Fortin PM, Bassett K, Wright JM. Blood pressure lowering efficacy of renin inhibitors for primary hypertension: a Cochrane systematic review. J Hum Hypertens 2009; 23(8):495-502.

82. Parving $\mathrm{HH}$, Persson F, Lewis JB, Lewis EJ, Hollenberg NK. Aliskiren combined with losartan in type 2 diabetes and nephropathy. $\mathrm{N}$ Engl J Med 2008;358(23):2433-2446.

83. Peixoto AJ, Orias M. Is there a role for direct renin inhibitors in chronic kidney disease? Curr Opin Nephrol Hypertens 2009; 18(5):397-403.

84. Persson F, Rossing P, Schjoedt KJ et al. Time course of the antiproteinuric and antihypertensive effects of direct renin inhibition in type 2 diabetes. Kidney Int 2008; 73(12):1419-1425.

85. Segura J, Ruilope LM. Should diuretics always be included as initial antihypertensive management in early-stage CKD? Curr Opin Nephrol Hypertens 2009; 18(5):392-396.

86. Wilcox CS. New insights into diuretic use in patients with chronic renal disease. J Am Soc Nephrol 2002; 13(3):798-805.

87. Bakris GL. Effects of diltiazem or lisinopril on massive proteinuria associated with diabetes mellitus. Ann Intern Med 1990; 112(9):707-708.

88. Bakris GL, Barnhill BW, Sadler R. Treatment of arterial hypertension in diabetic humans: importance of therapeutic selection. Kidney Int 1992; 41(4):912-919.

89. Bakris GL, Weir MR, Shanifar S, Zhang Z, Douglas J, van Dijk DJ, Brenner BM. Effects of blood pressure level on progression of diabetic nephropathy: results from the RENAAL study. Arch Intern Med, 2003. 163(13):1555-1565.

90. Koshy S, Bakris GL. Therapeutic approaches to achieve desired blood pressure goals: focus on calcium channel blockers. Cardiovasc Drugs Ther 2000; 14(3):295-301.

91. Bakris GL, Fonseca V, Katholi RE et al. Differential effects of beta-blockers on albuminuria in patients with type 2 diabetes. Hypertension 2005; 46(6):1309-1315.

92. Marin R, Gorostidi M, Fernandez-Vega F, AlvarezNavascues R. Systemic and glomerular hypertension and progression of chronic renal disease: the dilemma of nephrosclerosis. Kidney Int Suppl 2005; (99):S52-56.

93. Navaneethan SD, Nigwekar SU, Sehgal AR, Strippoli G F. Aldosterone antagonists for preventing the progression of chronic kidney disease. Cochrane Database Syst Rev 2009; (3):CD007004.

94. Agarwal R, Alborzi P, Satyan S, Light RP. Dry-weight reduction in hypertensive hemodialysis patients (DRIP): a randomized, controlled trial. Hypertension 2009; 53(3):500-507.

95. Horl MP, Horl WH. Dialysis: Normovolemia is a therapeutic target for hypertension. Nat Rev Nephrol 2009; $5(8): 438-439$. 